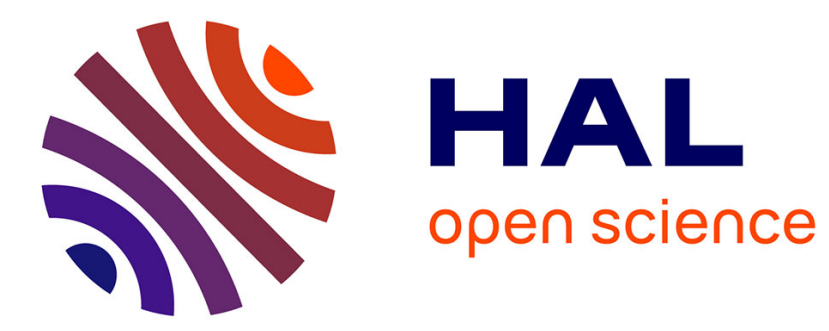

\title{
Demand response scheduling to support distribution networks operation using rolling multi-period optimization
}

\author{
Yujun He, Marc Petit
}

\section{- To cite this version:}

Yujun He, Marc Petit. Demand response scheduling to support distribution networks operation using rolling multi-period optimization. Journal of Process Control, 2019, 74, pp.13 - 22. 10.1016/j.jprocont.2018.07.005 . hal-01942621

\section{HAL Id: hal-01942621}

https://hal-centralesupelec.archives-ouvertes.fr/hal-01942621

Submitted on 22 Oct 2021

HAL is a multi-disciplinary open access archive for the deposit and dissemination of scientific research documents, whether they are published or not. The documents may come from teaching and research institutions in France or abroad, or from public or private research centers.
L'archive ouverte pluridisciplinaire HAL, est destinée au dépôt et à la diffusion de documents scientifiques de niveau recherche, publiés ou non, émanant des établissements d'enseignement et de recherche français ou étrangers, des laboratoires publics ou privés.

\section{(c) (1) $\$$}

Distributed under a Creative Commons Attribution - NonCommerciall 4.0 International 


\title{
Demand Response Scheduling to support Distribution Networks operation using Rolling Multi-period Optimization
}

\author{
Yujun $\mathrm{He}^{\mathrm{a}}$, Marc Petit ${ }^{\mathrm{a}} *$ \\ ${ }^{a}$ GeePs $\mid$ Group of Electrical Engineering Paris, UMR CNRS 8507, CentraleSupélec, Univ. Paris-Sud, UPMC, Gif-sur-Yvette, France
}

\begin{abstract}
This paper presents an approach of demand response (DR) scheduling with thermostatically controlled loads (TCL) in a distribution grid with high penetration of distributed generations (DG). In this approach, household TCL are employed as flexibility resources to support the distribution network for mitigating voltage or congestion constraints. A two-stage rolling optimization based control scheme is proposed to determine the optimal operating status of flexible loads using the forecast of generation and demand in the distribution system. The proposed methodology is conducted in a distribution test feeder with realistic scenarios. The simulation results have shown the usefulness and efficiency of the proposed method in improving the network operation and increasing the hosting capacity of DG.

Keywords: Demand response; distributed generation; distribution network; thermostatically controlled loads; rolling optimization.
\end{abstract}

\section{Introduction}

The growth of renewable energy sources (RES) and new demand such as battery electric vehicles (BEV), will introduce massive impacts on distribution network operation [1], such as voltage violation and congestion. In medium voltage (MV) networks the conventional voltage control approach using on-load tap changer (OTLC), based on the assumption of unidirectional power flow, is not designed for this change [2]. Additionally, in areas with large amount of RES and weak demand, some transformers at the connection between de transmission and distribution grids may be subjected to higher peak load during injection than during withdrawal from the transmission grid. Now distribution networks have to become (more) active to comply with the connection of large amount of DG. Demand response (DR) program is an attractive way to address this issue, as it can respond quickly with respect to the variation of DG [3]. Typically, most applications of DR are dedicated to the participation in the balancing markets or frequency regulation reserves because valorization markets still exist [4]. Nevertheless, many references integrate the use of flexibility in distribution grid operation [e.g. 5,6,17,18]. Demand response taking advantage of the thermal buffering capability of thermostatically controlled load (TCL) within residential buildings can be a flexibility methodology because buildings with high thermal inertia can absorb a remarkable amount of thermal energy for a shifted use. By dispatching the flexibility, the distribution system operator (DSO) can maximize the use of the current capacity of distribution system infrastructures and postpone investment for grid reinforcement. The optimal load dispatch issue in a distribution system can be divided into two sub-problems: how to optimize this part of load flow (e.g. flexible loads) in the distribution system to generate DR scheduling signal, and how to dispatch flexible loads in response to the DR scheduling signal without disturbing critically the comfort of end-users.

Many references intend to address the optimal power flow (OPF) problem which consists in managing diverse distributed energy resources (DER) in distribution systems. In [5], the load control signal is based on a real time OPF at a snapshot. The multi-period OPF accounting for the time series coupling of some DER is proposed in [7]. Due to the non-convex and nonlinear nature of the modeling of distribution system, the complexity of OPF increases remarkably as a function of the system scale. The solutions include linear approximations of the distribution system model [5] or a proposal for distributed algorithms to solve OPF separately [6]. Furthermore, an exact convex formulation of OPF can be found in $[8,9,10]$. In this approach, a semi-definite condition is adopted to relax the non-convex constraint in the model and formulate OPF as second order cone programming (SOCP). However, those references only study SOCP with a static view, so it is not able to tackle with the flexibility dispatch issue with time domain coupling. The SOCP based OPF in time-series can be found in [11][12]. In [11], the authors address a DR scheme using the SOCP based OPF in the day-ahead retail market. In [12], the SOCP based OPF is combined with a household energy management system and solved in a distributed way in order to keep the privacy of users. The stochastic method in this context has to be developed to consider uncertainties associated with generation and demand. 
From a bottom-up view, the flexibility dispatch is usually formulated as a decision making problem by operating the individual load status to minimize the overall costs of loads [14]. A detailed modeling of TCL in participating in demand response scheme can be found in [13]. The flexible load dispatch is formulated as a mixed integer linear programming (MILP) in [15]-[17]. In [17], a MILP formulation with detailed modeling of an aggregation of flexible loads is proposed for utility scale DR scheme, but the thermal appliances are not considered. In [20], the authors proposed a flexible demand model based on thermal appliances, and a formulation as a mixed integer non-linear programming (MINLP) for smart grid applications. In [18], a scheduling of electric vehicle charging is formulated in order to limit the impact on the distribution network in terms of power losses and voltage violation. In [19], the DR program with thermal and shiftable loads is used for alleviating distribution network congestions and cost savings for end-user. In [21], the nodal hourly pricing in day-ahead optimization framework of DR is proposed. The issue of flexibility dispatch is usually coupled with the generation of intermittent generation like wind turbine or PV in [22]-[24]. Due to the uncertainty of DG and demand, the day-ahead optimal scheduling might be inaccurate from the day-ahead forecast [25]. Probabilistic methods or stochastic optimization techniques can be employed as in [26]. The rolling optimization, also known as moving horizon optimization, is capable of solving real time decision making problem and proved to be effective [25, 27]. In [15], the load dispatch formulated as a MILP problem is solved by a rolling optimization based approach with a real time pricing policy. In [27], the charging schedule of electric vehicles (EV) is obtained from a rolling optimization approach considering the uncertain behavior of users.

Although there are quite a lot of researches studying these two aspects separately, few of them have considered the rolling optimization based flexibility dispatch that is integrated in a SOCP based OPF model. This paper proposes a rolling optimization based approach to dispatch the flexibility from household TCL in order to mitigate distribution grid constraints. The flexibility dispatch is formulated as a two-stage optimization: the first stage is to solve an OPF in a distribution network with a rolling horizon scheme; the second stage is to solve the flexible load dispatch at the aggregator level using a TCL model. The rolling optimization, which periodically updates input data information, is implemented in order to reduce the uncertainty associated with generation and consumption. The OPF problem is formulated using the branch load flow model considering conic relaxation. The SOCP based OPF is an exact convex formulation that can greatly reduce the complexity of network model while no approximation is introduced.

The major contributions of the proposed approach are that 1) it provides a two-stage optimization framework with SOCP-based OPF and detailed flexible load model to solve the flexibility dispatch problem in distribution system; and 2) it proposes a rolling robust optimization tool to tackle with the uncertainty associated with generation of RES and consumption. With a certain vision, the rolling optimization can effectively adjust forecasting errors and maximize the benefits from load scheduling.

This paper is structured as follows: the section 2 outlines system modeling including the flexibility scheme, the modeling of household flexible load, and distribution network model. The section 3 presents the rolling optimization based dispatch approach for flexible load. Then, the proposed approach is carried out on a realistic test distribution network described in the section 4 and simulation results are discussed in the section 5. Finally, conclusions and opened issues are drawn in the section 6.

\section{System Modeling}

\subsection{Architecture of household TCL management in the distribution grid}

Smart grids aim at making distribution networks with high penetration of DG more flexible to mitigate critical conditions including 1) large reverse power flow to the transmission system, 2) voltage constraints and 3) line congestions, for the purpose of postponing the grid reinforcement. The organization of household flexibility scheduling in a distribution network is illustrated in Fig.1. At first a state estimator detects network constraints and then a control center will operate OLTC, DG and sources of flexibility in a centralized way based on the current network state and forecasting of generation and consumption. A local flexible load aggregator is helpful in the management of household flexibility as most flexible loads are small-scaled and decentralized. The load aggregator can communicate with DSO the capabilities to increase or decrease the demand during a certain period and the associated costs. In addition, the load aggregator can offer a better load curve by smoothing the profiles from a massive number of flexible loads. As the intermediary between DSO and end-users, the load aggregator is might better negotiate with DSO for incentives.

In fact, the DSO needs for flexibility will depend on the location of the constraint. So the load aggregator has to control the flexible demand in a nodal approach. A similar structure of nodal DR management can be found in [28]. Therefore, some smart grid infrastructures are required to permit two-way communication between the load 
aggregator and flexible loads and enable the real-time control. The sources of household flexibility consist of load shedding, load shifting, and TCL. The second refers to devices that have to complete a prescribed energy requirement with a time limitation, such as electric vehicle charging or washing machine. The TCL refers to appliances with a thermal inertia. This paper only considers the energy management of TCL in residential buildings.

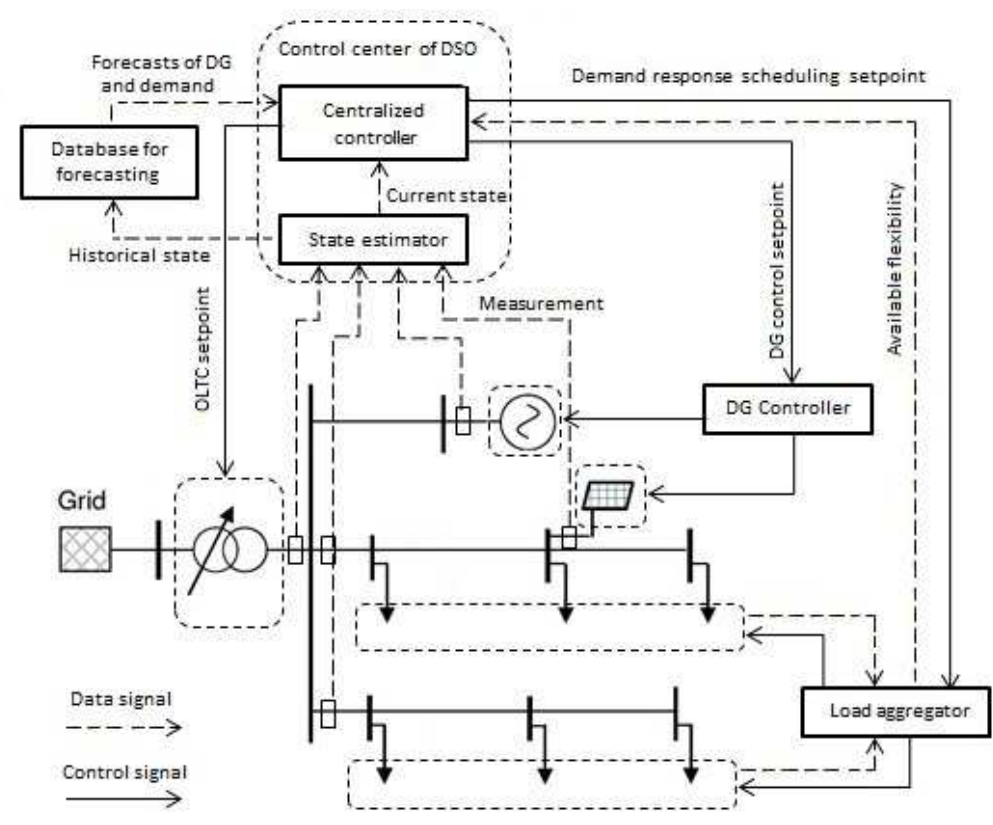

Fig. 1. Architecture of household TCL management in a distribution network

\subsection{Modeling of household TCL}

First, we introduce a household heating device (Fig 2), which is modeled as a first order system with a state variable $\theta_{i n}(t)$ denoting the indoor temperature and a binary control variable $P_{i n}(t)$ denoting the electric power. $R_{t h}$ and $C_{t h}$ are respectively the thermal resistance and thermal capacity of the room. The ambient temperature $\theta_{0}(t)$ depends on the external environment. $P_{\text {in }}(t)$ is switched from 0 to $P_{\text {rate }}$ (and inversely) to keep $\theta_{\text {in }}(t)$ within the temperature ranges $\left[\theta_{\min }, \theta_{\max }\right]$. The dynamics of $\theta_{i n}(t)$ in $(1)$ can be found in [30], where $P_{\text {rate }}$ is the rated power of appliance. The detailed parameters are presented in table 1.

$$
\dot{\theta}_{i n}(t)=\frac{1}{R_{t h} C_{t h}}\left(\theta_{0}(t)-\theta_{i n}(t)\right)+\frac{1}{C_{t h}} \cdot P_{i n}(t)
$$

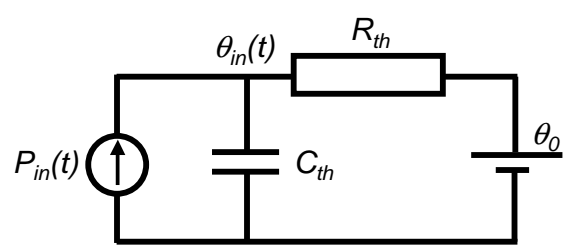

Fig. 2. Equivalent thermal circuit scheme of a household heating device

In this paper, we are interested in the nodal flexibility in a MV distribution network. Hereafter the nodal flexibility of thermal appliances is presented by an aggregate model of $N$ heating devices with homogenous characteristics located behind a MV/LV transformer. Let $N$ denote the number of appliances at the bus, $\boldsymbol{P}_{\text {in }}(t)$ and $\boldsymbol{\theta}_{i n}(t)$ denote the vector of $P_{i n}(t)$ and $\theta_{i n}(t)$ of $N$ appliances. We define the average internal temperature $\theta_{a v}(t)=\Sigma \boldsymbol{\theta}_{i n}(t) / N$ and aggregate power $P_{a g g}(t)=\Sigma \boldsymbol{P}_{i n}(t)$ as two output variables of the aggregate load model. Thus, this aggregate model can be expressed in the state-space representation in (2), where $A, B, C, D$ are matrices that can be computed from (1). 


$$
\left\{\begin{array}{c}
\dot{\boldsymbol{\theta}}_{i n}(t)=A \cdot \boldsymbol{\theta}_{i n}(t)+B \cdot\left[\begin{array}{l}
\boldsymbol{P}_{i n}(t) \\
\theta_{0}(t)
\end{array}\right] \\
{\left[\begin{array}{c}
P_{a g g}(t) \\
\boldsymbol{\theta}_{a v}(t)
\end{array}\right]=C \cdot \boldsymbol{\theta}_{i n}(t)+D \cdot\left[\begin{array}{l}
\boldsymbol{P}_{i n}(t) \\
\boldsymbol{\theta}_{0}(t)
\end{array}\right]}
\end{array}\right.
$$

It can be demonstrated that this system is observable and controllable. Anyhow, the control of this aggregate model could be realized for the given output $P_{a g g}(t)$ and $\theta_{a v}(t)$ at the moment $t$ but the detailed control strategy is not in the scope of this paper. Hereafter, we focus on seeking the optimal setpoint of $P_{a g g}(t)$ and $\theta_{a v}(t)$ as they determine the available flexibility of the aggregate TCLs. The evolution of aggregate load model for the timeslot $t$ is obtained by summing up the dynamics in (1) and transformed to a discrete format with a time step $\Delta t$.

$$
\theta_{a v, t+1}=\left(1-\frac{\Delta t}{R_{t h} C_{t h}}\right) \cdot \theta_{a v, t}+\frac{\Delta t \cdot \theta_{0, t}}{R_{t h} C_{t h}}+\frac{\Delta t \cdot P_{a g g, t}}{N C_{t h}}
$$

From (3), the evolution of aggregate model can be determined with the setpoint of $P_{a g g}(t)$ and $\theta_{a v}(t)$ and the environment variable $\theta_{0}(t) . P_{\text {agg }}(t)$ can be varied continuously within $\left[0, N P_{\text {rate }}\right]$ as long as $N$ is large enough. $\theta_{a v}(t)$ is limited within $\left[\theta_{\text {min }}, \theta_{\text {max }}\right]$, because it is a necessary condition for limiting all the variables of $\boldsymbol{\theta}_{\text {in }}(t)$ within this range. The setpoint of $\theta_{a v}(t)$ is preset to $\theta_{\text {ref }}=\left(\theta_{\min }+\theta_{\max }\right) / 2$. A daily power demand profile of this aggregate load model with a typical ambient temperature pattern is shown in Fig. 3 . The detailed parameters of heating advice can be found in table 1 (issued from [30]).

Table 1. Parameters of thermal appliances in the simulation.

\begin{tabular}{ll}
\hline Parameter & Value \\
\hline$R_{t h}($ Thermal resistance $)$ & $7.5^{\circ} \mathrm{C} / \mathrm{kW}$ \\
$C_{t h}($ Thermal capacity $)$ & $2.19 \mathrm{kWh} /{ }^{\circ} \mathrm{C}$ \\
$\theta_{\min }($ Minimum internal temperature $)$ & $17.3^{\circ} \mathrm{C}$ \\
$\theta_{\max }($ Maximum internal temperature $)$ & $19.3^{\circ} \mathrm{C}$ \\
$\theta_{\text {ref }}($ Reference indoor temperature $)$ & $18.3^{\circ} \mathrm{C}$ \\
$\theta_{0}($ Ambient temperature $)$ & $6 \sim 16^{\circ} \mathrm{C}$ \\
$P_{\text {rate }}($ Rated power $)$ & $3 \mathrm{~kW}$ \\
\hline
\end{tabular}

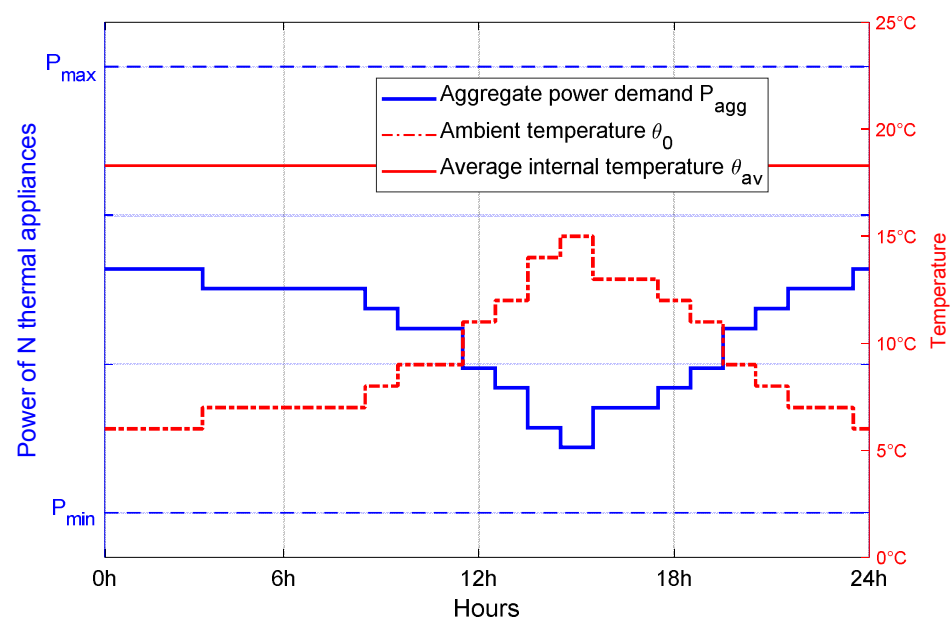

Fig. 3. 24hrs profiles of $P_{a g g}(t)$ and $\theta_{a v}(t)$ with a daily ambient temperature profile

It can be seen that the power demand $P_{a g g}(t)$ is influenced by the ambient temperature $\theta_{0}(t)$. With higher ambient temperature, the heating advices consume less electric energy for keeping the indoor temperature, and vice versa. This can be used to model the dynamics of homogeneous loads. When including heterogeneous loads, we can partition them using clustering methods according to their parameters and model them separately, which would be studied in future work. 


\subsection{Modeling of distribution network}

The OPF in distribution networks is formulated using a branch flow model introduced in [10]. Let $\mathcal{B}$ denote the set of busses $(i)$ and $\mathcal{L}$ denote the set of branches $(i, j)$. For each branch $(i, j)$, let $z_{i j, t}=r_{i j, t}+i x_{i j, t}$ denote the complex impedance of the branch, $I_{i j, t}$ denote the complex current and $S_{i j, t}=P_{i j, t}+i Q_{i j, t}$ denote the complex power flow from buses $i$ to $j$ at time slot $t$. For each bus $(i), V_{i, t}$ denote the complex voltage and $S_{i, t}=P_{i, t}+i Q_{i, t}$ denote the complex bus load.

The whole power demand at bus $i$ is divided into three components: unflexible demand, flexible demand (which is modeled in subsection 2.2), and DG connected at this bus, as shown in (4). The unflexible demand doesn't participate in the demand response program and its power profile comes from a public database of Enedis (the main DSO in France) for the region of Provence in France.

$$
\left\{\begin{array}{c}
P_{i, t}=P_{u n, i, t}+P_{a g g, i, t}-P_{D G, i, t} \\
Q_{i, t}=Q_{u n, i, t}+Q_{a g g, i, t}-Q_{D G, i, t}
\end{array}\right.
$$

The electric circuit characteristics of the radial distribution network are presented by the following equations:

$$
\begin{aligned}
& V_{i, t}-V_{j, t}=z_{i j} I_{i j, t} \\
& S_{i j, t}=V_{i ; t} I_{i j, t}^{*} \\
& S_{i j, t}-z_{i j} z_{i j}\left|I_{i j, t}\right|^{2}-\sum_{(j ; k) \in \mathcal{L}} S_{j k, t}=s_{j, t}
\end{aligned}
$$

By expressing (5)-(7) in terms of real variables, it becomes:

$$
\begin{aligned}
& P_{i j, t}-r_{i j} l_{i j, t}-\sum_{(j ; k) \in \mathcal{L}} P_{j k, t}=P_{j, t} \\
& Q_{i j, t}-x_{i j} l_{i j, t}-\sum_{(j ; k) \in \mathcal{L}} Q_{j k, t}=Q_{j, t} \\
& v_{j, t}=v_{i, t}-2\left(r_{i j} P_{i j, t}+x_{i j} Q_{i j, t}\right)+\left(r_{i j}^{2}+x_{i j}^{2}\right) \cdot l_{i j, t} \\
& l_{i j, t}=\frac{P_{i j, t}^{2}+Q_{i j, t}^{2}}{v_{i, t}}
\end{aligned}
$$

where $l_{i j, t}=\left|I_{i j, t}\right|^{2}$ and $v_{i, t}=\left|V_{i, t}\right|^{2}$.

The computational burden is considerable due to the non-convex nature of the described network model. Thus, a semi-definite relaxation of (11) to a convex condition is implemented in order to formulate a SOCP [10].

$$
l_{i j, t} \geq \frac{P_{i j, t}^{2}+Q_{i j, t}^{2}}{v_{i, t}}
$$

The HV/MV transformer with on load tap changer (OLTC) can be switched for keeping the voltage magnitude at lower side within a preset dead band. In this paper an ideal equivalent transformer model is used to adjust the voltage at slack bus (13), and the tap position must be within limits (14).

$$
\begin{aligned}
& v_{\text {slack }, t}=n\left(\operatorname{Tap}_{t}\right)^{2} v_{\text {slack } 0} \\
& \operatorname{Tap}_{\text {min }} \leq \operatorname{Tap}_{t} \leq \operatorname{Tap}_{\text {max }}
\end{aligned}
$$

Besides, the bus voltage and branch apparent power must be kept within system secure constraints (15) and (16). 


$$
\begin{aligned}
& V_{\min }^{2} \leq v_{i, t} \leq V_{\max }^{2} \\
& P_{i j, t}^{2}+Q_{i j, t}^{2} \leq S_{\max }^{2}
\end{aligned}
$$

The DG connected in the distribution network includes wind turbines (WT) and roof-top PV. It is assumed that the maximum power point tracking (MPPT) technique is used to determine maximum active power $\boldsymbol{P}_{D G, i, t}^{\max }$ from RES at timeslot $t$ in (17), where $\lambda_{i, t}$ depends on weather conditions (e.g. wind speed and solar insolation).

$$
P_{D G, i, t}^{\max }=\lambda_{i, t} P_{D G, i}^{\text {install }}
$$

The active and reactive power regulation of DG at the bus $i$ is expressed in (18)-(20).

$$
\begin{aligned}
& 0 \leq P_{D G, i, t} \leq P_{D G, i, t}^{\max } \\
& Q_{D G, i, t}^{\min } \leq Q_{D G, i, t} \leq Q_{D G, i, t}^{\max } \\
& P_{D G, i, t}^{2}+Q_{D G, i, t}^{2} \leq S_{D G, i}^{\max ^{2}}
\end{aligned}
$$

\section{Rolling optimization formulation for TCL scheduling}

The rolling optimization is an effective approach to make real time decision of the load dispatch. The procedures of rolling optimization of flexible load dispatch are described as follows:

1) The total scheduling horizon from 0 to $T$ is determined as 24 hours of a day.

2) At the first time step, compute the OPF over the forecasting horizon $\tau$ and generate a set of scheduling of flexible load. Only the scheduling at real time period $t_{0}$ is ever implemented.

3) At each time step, move one step ahead the forecasting horizon and repeat the step 2) until the end of the day based on current operational status and updated information and generate the a new set of load dispatch. It is assumed in this work that new forecasts are available every $30 \mathrm{~min}$.

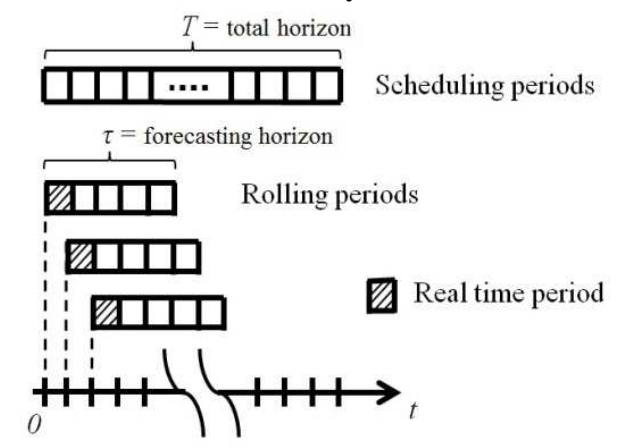

Fig. 4. Procedures of rolling optimization

At each real time period $\mathrm{t}_{0}$, the rolling optimization is formulated in (21).

$$
\begin{aligned}
& \min _{x_{t}}\left(F\left(x_{t_{0}}\right)+\sum_{t=t_{0}+1}^{t_{0}+\tau} \hat{F}\left(x_{t}\right)\right), \\
& \text { s.t. } \quad G\left(x_{t}\right) \leq 0, \quad t \in\left[t_{0}, t_{0}+\tau\right]
\end{aligned}
$$

where $F\left(x_{t_{0}}\right)$ is the objective at the current timeslot and $\hat{F}\left(x_{t}\right)$ the forecast of objective at future timeslots, $G\left(x_{t}\right)$ is the set of constraints at each timeslot.

The detailed formulations at distribution network level and load aggregator level are presented as follows. 


\subsection{Stage of optimization at distribution network level}

The OPF for distribution network in this paper aims at minimizing power losses in the feeder while maximizing the generation from RES subject to system secure constraints.

Firstly a load flow calculation is run within the optimization window $\left[t_{0}, t_{0}+\tau\right]$ according to the demand and RES generation forecastings. From these results, the set of timeslots $\mathcal{T}_{c r t}$ are identified during which there are critical conditions on the network that are due to RES or peak loads.

Then the OPF is carried out to determine the setpoint of DG, OLTC, and DR at each timeslot. The objective function of OPF to be minimized is composed of power losses on the lines, DG curtailment and reverse active power at the slack bus for each timeslot $t$ as expressed in (22).

$$
\begin{gathered}
\min \left(c_{1} \sum_{(i, j)} r_{i j} \cdot l_{i j, t}+c_{2} \sum_{k}\left(P_{D G, k, t}^{\max }-P_{D G, k, t}\right)+c_{3}\left|-P_{\text {slack }, t}\right|^{+}\right), \\
(i, j) \in \mathcal{L}, k \in G, t \in\left[t_{0}, t_{0}+\tau\right]
\end{gathered}
$$

where $G$ is the set of busses with a dispatchable DG, the notation $|x|^{+}=x$ if $x \geq 0,|x|^{+}=0$ if $x<0 ; P_{\text {slack,t }}$ is the active power import at slack bus and negative value of $P_{\text {slack,t }}$ means the electrical power is fed back towards the higher voltage grid; $c_{1}, c_{2}$ and $c_{3}$ are weighting coefficients of each objective. In this study, the choice of the weighting factors is identical, i.e. $c_{1}=c_{2}=c_{3}=1 / 3$.

The OPF is subjected to the network equality and inequality constraints including (4), (8), (9), (10), (12), (13), (14), (15), (16), (17), (18), (19), and (20). It is noticed that the control variable of DR $P_{a g g, i, t}$ appears in the equation (3).

Let $\boldsymbol{P}_{a g g, i}^{r e f}$ denote the vector $P_{a g g}(t)$ at bus $i$ obtained from the results of OPF. $\boldsymbol{P}_{a g g, i}^{r e f}$ will be used as a reference of the optimization at load aggregator. In fact, the need of flexibility for DSO in terms of DR duration and magnitude has been modulated in $\mathcal{T}_{c r t}$ and $\boldsymbol{P}_{a g g, i}^{r e f}$. Then the DSO sends $\mathcal{T}_{c r t}$ and $\boldsymbol{P}_{a g g, i}^{r e f}$ of each bus to the load aggregators for determining the DR scheduling at bus $i$.

\subsection{Stage of optimization at load aggregator level}

The optimization run by a load aggregator is targeted to keep the temperature comfort of thermal appliances and respond to the DSO need for flexibility. As described in the previous section, the control of thermal appliances can be realized with the setpoint $P_{a g g}(t)$ and $\theta_{a v}(t)$. Let $\boldsymbol{P}_{a g g, i}$ and $\boldsymbol{\theta}_{a v, i}$ denote respectively the vector of $P_{a g g}(t)$ and $\theta_{a v}(t)$ over the optimization window at bus $i$. The objective function of DR aims at minimizing the power deviation from the setpoint of OPF during timeslots of $\mathcal{T}_{c r t}$ and minimizing the influence on the temperature comfort during the other timeslots, as expressed in (23):

$$
\begin{aligned}
& \min \left(\boldsymbol{a}_{1}^{T} \cdot\left\|\boldsymbol{P}_{a g g, i}-\boldsymbol{P}_{a g g, i}^{r e f}\right\|^{2}+\left(1-\boldsymbol{a}_{1}^{T}\right) \cdot\left\|\boldsymbol{\theta}_{a v, i}-\theta_{s e t}\right\|^{2}\right), i \in \mathcal{B} \\
& a_{1}(t)=1 \text { if } t \in \mathcal{T}_{c r t} \text { and } a_{1}(t)=0 \text { if } t \notin \mathcal{T}_{u n}, t \in\left[t_{0}, t_{0}+\tau\right]
\end{aligned}
$$

where $\mathbf{a}_{1}$ is a $\tau$-element vector and $a_{1}(t)$ is the element of $\mathbf{a}_{1}$.

The optimization of thermal appliances is subject to the equality constraint in (3) as well as the inequality constraints in (24) and (25).

$$
\begin{aligned}
& \theta_{\min } \leq \boldsymbol{\theta}_{a v, i} \leq \theta_{\max } \\
& P_{a g g, i}^{\min } \leq \boldsymbol{P}_{a g g, i} \leq P_{a g g, i}^{\max }
\end{aligned}
$$

The lower and upper limits $P_{a g g, i}^{\min }$ and $P_{a g g, i}^{\max }$ are determined by the number of thermal appliances $N_{i}$ at bus $i$, i.e. $P_{a g g, i}^{\min }=0$ and $P_{a g g, i}^{\max }=N_{i} P_{\text {rate }}$. The optimal setpoint of DR is denoted $\boldsymbol{P}_{a g g, i}^{\text {opt }}$ and $\boldsymbol{\theta}_{a v, i}^{o p t}$. It is noticed that there might be some deviations between $\boldsymbol{P}_{a g g, i}^{\text {ref }}$ and $\boldsymbol{P}_{a g g, i}^{\text {opt }}$ due to operational constraints of DR. Therefore, the DSO has to relaunch the OPF using a fixed value $\boldsymbol{P}_{a g g, i}^{\text {opt }}$ from optimization of DR to obtain the new optimal setpoint of DG. 


\subsection{Flow chart of proposed DR scheduling}

The procedure of DR scheduling is organized in Fig. 5. The rolling optimization based control runs for each timeslot over the horizon $T$. At each timeslot $t_{0}$, the optimization window covers $\left[t_{0}, t_{0}+\tau\right]$. The stages of optimization on the level of distribution network and load aggregator are carried out consecutively to obtain the vector of control variables $\boldsymbol{P}_{a g g, i}^{o p t}$ and $\boldsymbol{\theta}_{a v, i}^{o p t}$. Only the optimal value of DR at the first timeslot $t_{0}$ is implemented. The optimal value of DG regulation obtained at timeslot $t_{0}$ has to be renewed with the output of load aggregator before being used by their local controller.

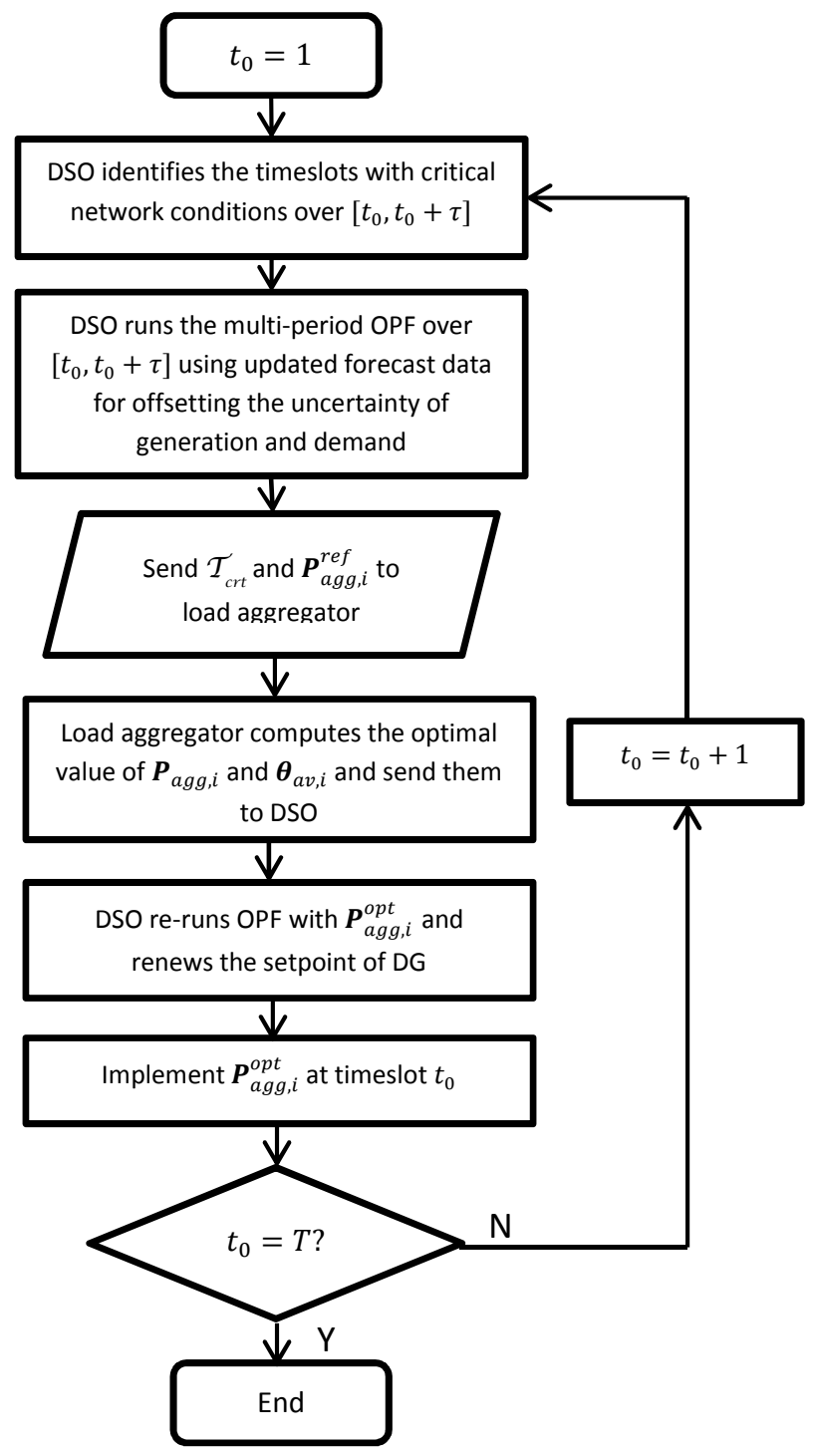

Fig. 5. Flow chart of proposed DR scheduling

\section{Case Study}

\subsection{Test distribution network setting}

To demonstrate the usefulness and effectiveness of proposed method, we perform simulation using an IEEE 34-bus test feeder in Fig. 6 with several modifications. Typical values of the French network have been used for cabled lines and overhead lines parameters. The total demand on the feeder is up to $9.52 \mathrm{MW}$. The detailed characteristics of this test network can be found in [29]. The RES connected in this feeder include WT and rooftop PV. We assume two WTs, each with installed power of 4MW, are connected within the feeder and controllable through an agreement between the DSO and the WT operator. The reactive power of WT is 
dispatched within $\left[-0.35 \cdot P_{D G, i}^{\text {install }}, 0.4 \cdot P_{D G, i}^{\text {install }}\right]$ to offer voltage support. The installed power of roof-top PV connected at each bus is sized as a function of MV/LV transformer capacity and the PV is non-dispatchable. The overall demand at each bus is shared between unflexible demand (70\%) and flexible demand (30\%).

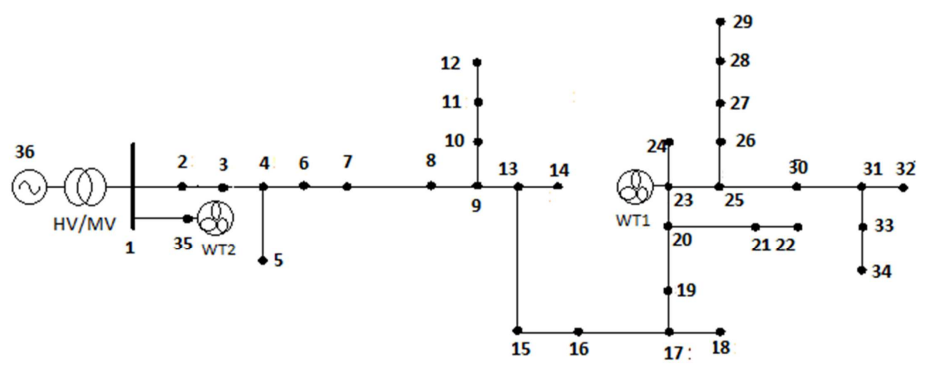

Fig. 6. Test distribution network

The OLTC has 17 tap positions to vary the transformer voltage from $-10 \%$ to $+10 \%$, so with each change of tap position the voltage variation is $1.25 \%=0.0125 \mathrm{pu}$. The OLTC is set to keep the bus voltage at substation within [1.02pu, 1.04pu], which is commonly used by French DSO.

\subsection{Simulation scenarios}

The forecasting patterns of unflexible demand, wind and PV generations are shown in Fig. 7. It can be seen that during 13-17h, there is reverse power flow to the transmission system due to the large penetration of RES. The temperature pattern used in the simulation has been shown in Fig.3. The time step is set to $\Delta t=30 \mathrm{~min}$, so the total horizon $T=48$ timeslots for the whole day simulation. A forecasting window of $6 \mathrm{~h}$ which is equal to 12 timeslots is adopted in the rolling optimization.

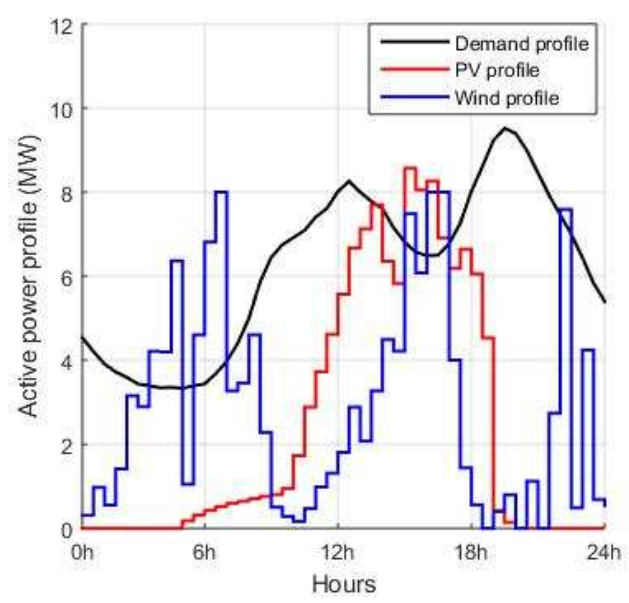

Fig. 7. 24hrs forecasting patterns of demand, PV and WT on the test feeder.

The simulations are conducted in four scenarios to demonstrate the effectiveness and efficiency of proposed method as follows:

- Scenario 1, the base case where the flexible loads are not controlled in the network operation;

- Scenario 2, the flexible loads are controlled using rolling optimization approach but the uncertainty of demand and generation are not taken into account, i.e. we suppose that the measured value ("real value") is exactly the predict value;

- Scenario 3, the flexible loads are controlled using rolling optimization approach and the uncertainty of demand and generation are taken into account, i.e. we suppose the measured value is deviated from the forecasted value, and the forecast errors follow a normal distribution;

- Scenario 4, the flexible loads are controlled using $24 \mathrm{~h}$ off-line information of demand and generation without forecast errors, like a day-ahead scheduling.

The prediction and optimization horizon in scenarios 2 and 3 is $6 \mathrm{~h}$. We suppose the prediction errors in scenario 3 are described by a normal distribution $N(1,0.1)$. The settings of these contrastive scenarios aim at 
verifying the robustness of proposed method against forecast errors and the length of optimization horizon.

\section{Simulation Results and Discussions}

The simulations are performed using MATLAB and the load flow is computed using the toolbox MATPOWER 5.0. First we compare the results of scenarios 1 and 2, respectively without and with DR scheduling. The demand and generation patterns in Fig. 8 implicate a large amount of reverse power flow towards the HV grid during the time intervals $5 \mathrm{~h} \sim 7 \mathrm{~h}$ and $13 \mathrm{~h} \sim 17 \mathrm{~h}$. In the conventional market structure, the reverse power flow to the HV grid is unlikely to be refunded. Thus, the DR scheduling is increasing the demand to absorb a part of this production, and thus to reduce the excess of local generation. It can be found that in the proposed method the demand shifting can be done after and before the peak of generation. This is because the rolling optimization is able to anticipate the need of flexibility using prediction data. The level of flexibility is limited by the operational constraints of flexible loads, which will be presented lately.

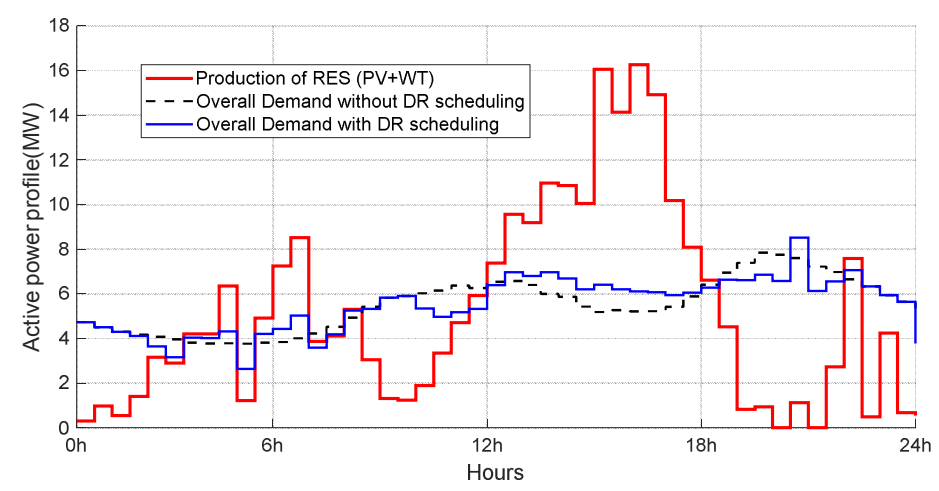

Fig. 8. 24hrs of demand and RES production profiles

Then, the voltage profiles at two critical hours $16 \mathrm{~h}$ and $19 \mathrm{~h} 30$, respectively representing the hours with full production and peak demand hours, are shown in Fig. 9. At 16h, both WT and PV are around their maximum power production and the voltage profile could exceed the upper limit at some busses. The reactive power regulation of WT is used to offer voltage support as shown in Fig.10, but it is not enough to mitigate the voltage deviations (this is due to the low $x / r$ ratio of MV lines). The absorption of reactive power of WT1 and WT2 during $15 \sim 17 \mathrm{~h}$ is for mitigating the voltage rise due to DG. At other hours, the WT1 injects reactive power for supporting the voltage profile as high as possible to reduce the power losses. As the reactive power regulation is not efficient enough to keep voltage inside the limits, the proposed method is able to remove the voltage constraint by both reducing the active power of DG and filling the valley of demand. In this example, the DR action helps to decrease the active power reduction of WT1 at $16 \mathrm{~h}$ by $0.20 \mathrm{MW}$. Thus, the rate of use of RES is improved with the use of DR. The DR scheduling can also remove low voltage constraints at $19 \mathrm{~h} 30$ by shifting the demand to other hours. Although congestions are not observed in this feeder, it could be obviously solved by shifting the flexible demand as well.

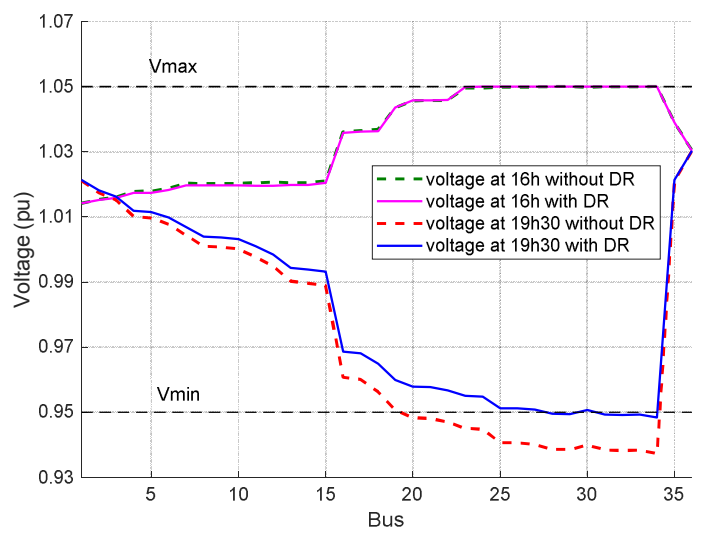

Fig. 9. Voltage profiles at $16 \mathrm{~h}$ and $19 \mathrm{~h} 30$. 

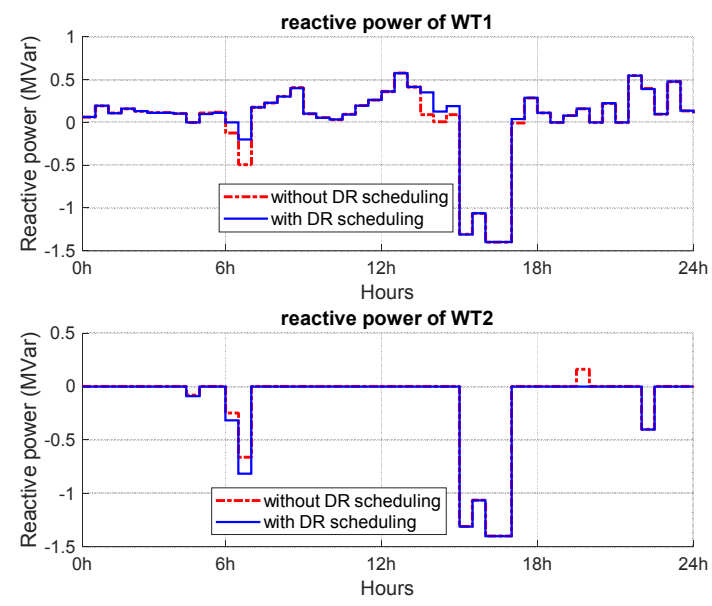

Fig.10. Reactive power regulation of WT generation (reactive power is positive if delivered into the grid)

The optimal setpoint $\boldsymbol{P}_{a g g, i}^{o p t}$ of DR scheduling is shown in Fig. 11. As the DR scheduling works in a nodal approach, we only display the profiles at the busses 8 and 24 that are respectively upstream and downstream the WT1. The proposed method has shifted the demand of thermal appliance from morning peak period $(10 \mathrm{~h} \sim 12 \mathrm{~h})$ to afternoon off-peak period (13h 17h). As it is not enough to mitigate the voltage constraints, the demand during $19 \mathrm{~h} \sim 20 \mathrm{~h} 30$ is also partially shifted before $19 \mathrm{~h}$ (anticipation of the heating with an increase of internal temperature before 19h). It is noticed that the flexible demand at bus 24 shows intensive solicitations in the $4 \mathrm{~h} \sim 8 \mathrm{~h}$ period. This is because the downstream busses are with greater voltage sensitivity than the upstream busses, so the use of their flexibility is more excessive which results in some oscillations of thermal loads and CLPU effect. For avoiding this situation, the use of flexibility should be shared among busses in a more moderate way, for example by applying solicitation limits for DR at each bus. The internal temperature profiles of thermal appliances at busses 8 and 24 are shown in Fig. 12. The internal temperature of thermal appliances is always kept within the limits to ensure the comfort of users who allow their devices participation in the grid operation. It can be found from the action of DR that the temperature constraint (then the users comfort) is the strongest condition to impede the use of thermal appliance arbitrarily.
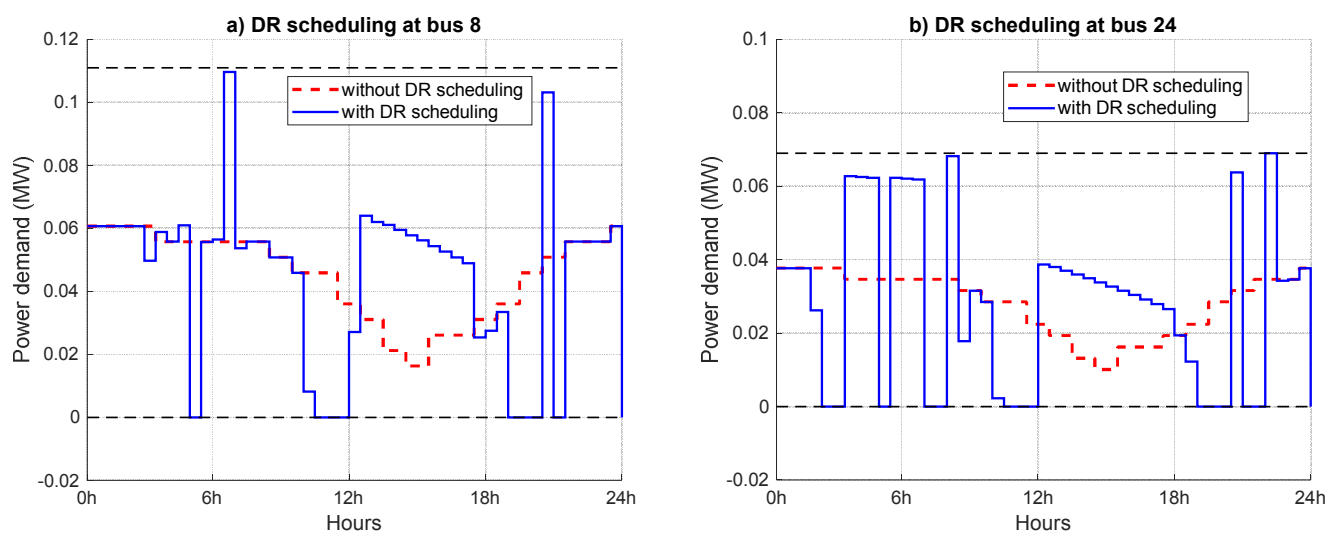

Fig. 11. Power profile $\boldsymbol{P}_{a g g, i}^{\text {opt }}$ of DR scheduling a) at bus 8, b) at bus 24. 

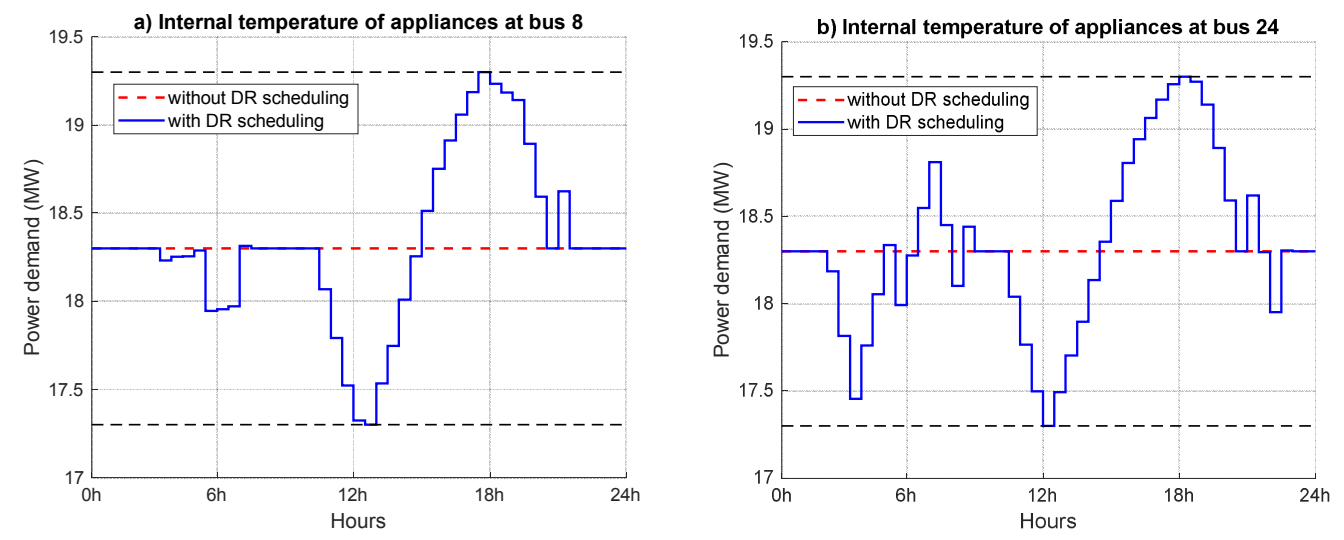

Fig. 12. Internal temperature $\boldsymbol{\theta}_{a v, i}^{o p t}$ of DR scheduling a) at bus 8, b) at bus 24 .

The detailed results of four scenarios are presented in table 2. It is shown that all performance indicators including power losses, DG curtailment and reverse power flow in the scenarios 2, 3 and 4 have been improved in presence of DR scheduling. For the single day that was studied, the energy injected into the HV grid is respectively reduced by $11.8 \%, 10.6 \%$ and $12.0 \%$ and the energy savings are respectively $12.2 \%, 11 \%$ and $12.3 \%$. The energy savings, i.e. the energy withdrawn from the HV grid, are constituted by the minimization of power losses, the reduction of DG curtailment, and the shifting of demand. This performance is limited by the TCLs as their temperature constraints are always respected. The overall demand of TCL is almost constant in the four scenarios. The results have shown that the performances of scenarios 2 and 3 are similar, and are close to scenario 4. Thus, it confirms the effectiveness of the proposed rolling optimization with respect to the off-line optimization. In particular, the results of scenario 3 compared with those of scenario 2 have proven the robustness of proposed method against the uncertainty of production of DG. This is because the optimal status of DR is adjusted when the rolling optimization is performed with updated forecasting of RES at each step. Thus, it is demonstrated that the proposed method can be implemented in a realistic situation considering the uncertainty of forecast. In addition, the line losses are always reduced in scenarios 2, 3 and 4 because the objective function of OPF takes them into account.

Table 2. Performance of network operation with DR for a single day. Total generation by DGs: 124.3 MWh

\begin{tabular}{|c|c|c|c|c|}
\hline & Scenario 1 & Scenario 2 & Scenario 3 & Scenario 4 \\
\hline Daily line losses & $2.87 \mathrm{MWh}$ & 2.60MWh & $2.62 \mathrm{MWh}$ & $2.59 \mathrm{MWh}$ \\
\hline Energy of active power curtailment of DG & $1.55 \mathrm{MWh}$ & $0.87 \mathrm{MWh}$ & $0.90 \mathrm{MWh}$ & $0.86 \mathrm{MWh}$ \\
\hline Energy injected into the HV grid & 38.03MWh & $33.55 \mathrm{MWh}$ & 33.98MWh & 33.47MWh \\
\hline Energy withdrawn from the HV grid & $50.76 \mathrm{MWh}$ & 44.57MWh & 45.19MWh & $44.53 \mathrm{MWh}$ \\
\hline Energy saving by DR & - & $6.19 \mathrm{MWh}$ & $5.57 \mathrm{MWh}$ & $6.23 \mathrm{MWh}$ \\
\hline Total flexible demand of TCL & $28.23 \mathrm{MWh}$ & 27.49MWh & $27.63 \mathrm{MWh}$ & $27.55 \mathrm{MWh}$ \\
\hline
\end{tabular}

Furthermore, a sensitivity analysis is conducted with different penetration of DG and flexibilities to verify the performance of proposed method. First the installed capacity of WT1 and WT2 is varied from 1 to 6MW for each WT. We use the energy savings by DR as the indicator to assess the performance of DR. In Fig. 13, the energy saving on the distribution network increases almost linearly with installed power capacity. The influence of available flexible demand in the feeder is also investigated in the sensitivity analysis. Three percentages of flexible demand are used, respectively $15 \%, 30 \%$ and $50 \%$. The percentage of energy savings as indicator is shown in Fig.14. It is indicated that the capabilities of DR is increasing when it takes greater part in the overall demand. The rolling optimization in scenario 2 can reach the performance of an off-line optimization with full information (scenario 4), while it requires less forecasting window for the production of DG. The robustness of proposed method under prediction errors is also assessed as the indicator of scenario 3 is always comparably close to the optimal results when WT keeps increasing. 


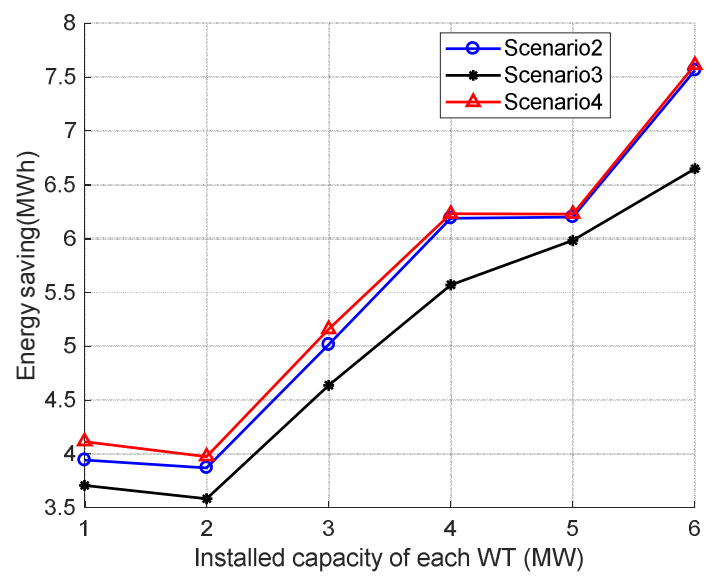

Fig. 13. Energy saving with DR as a function of installed capacity of WT.

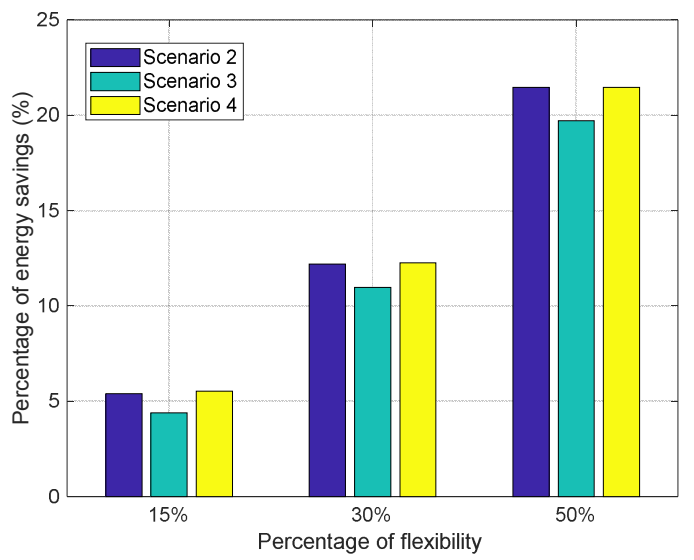

Fig. 14. Energy saving for different available percentage of flexibility

Then, the influences of forecast error and the length of prediction horizon $\tau$ are taken into consideration. The length of window is varied from $1 \mathrm{~h}$ to $8 \mathrm{~h}$ to test how the predictive vision can influence the optimization. Three levels of forecast errors, respectively normal distribution $N(1,0.05), N(1,0.1)$ and $N(1,0.2)$, are used to compare with the scenario 2. In order to smooth the fluctuations due to randomness, a mean value of indicator for 10 realizations is used. The indicator of energy saving as a function of the prediction horizon $\tau$ with these error settings is presented in Fig.15. It is shown that the performance of DR can be improved in increasing the window $\tau$ and the forecast accuracy. This is because the proposed method can better anticipate the use of flexibility with a longer window. The curve without forecast error indicates that the capabilities of flexibility are almost saturated when $\tau$ reaches $6 \mathrm{~h}$. It is also shown that the forecast error leads greater influence with longer window, since the uncertainties related to the rolling optimization is greater. 


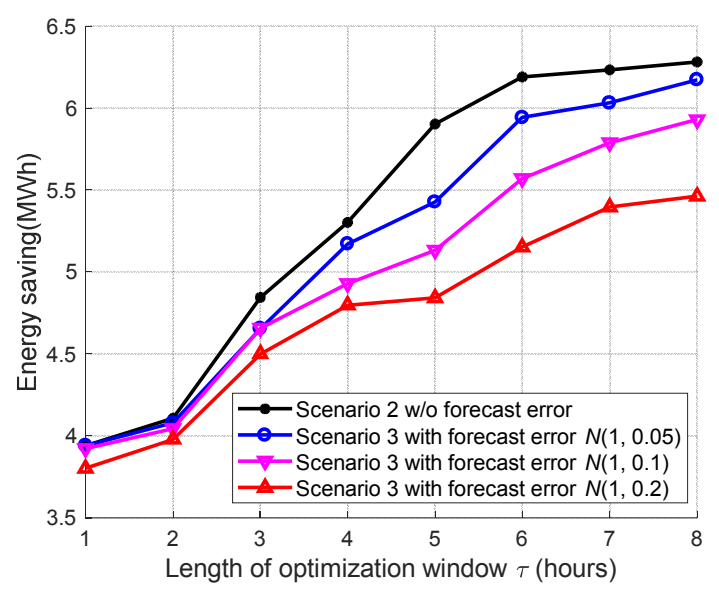

Fig. 15. Energy saving for different forecast horizon $\tau$

\section{Conclusions}

The DR scheduling for thermal appliances using a rolling multi-period optimization based control has been proposed for the operation of a MV distribution network with high penetration of DG. The approach is based on the forecasts of the production and demand, and the availability of the flexible demand. The DR scheduling can be used to mitigate the voltage and congestion constraints when a great amount of DG is connected, and reduce the power losses in the distribution network. The DR will also help the DSO to respect its forecasted power profile with the transmission system operator (TSO) at the HV/MV substation. The overall profit in the distribution feeder is improved as the energy import from HV grid is reduced. The DSO will then be able to optimize its contracted power with the TSO. The DR will also help the DSO in postponing expensive reinforcements (building of a new substation, new feeders, and new transformer). Finally, the operational constraints of temperature for the TCLs are always respected in the proposed method, so the comfort of endusers is not seriously disturbed.

The proposed method is conducted in a test distribution feeder with high penetration of DG that has critical reverse power flow and voltage deviations. The prediction uncertainty of production and demand is taken into account. The results have shown that the proposed method can effectively employ the flexible loads to support the distribution network operation. The robustness of the proposed method is assessed against the uncertainty related to the forecasted data. Although the results may depend on the specific feeder characteristics, this paper proposes a methodology of DR scheduling for the distribution network optimization. Furthermore, the modeling of household TCL is based on the homogenous assumption. The future work for heterogeneous loads can be done using some innovative energy management approaches, and by developing a more detailed aggregated model of the flexible loads at the MV/LV substation level. Finally, the scheduling of flexibility is based on technical issues (smart devices to control the loads), the socio-economic assessment (consumer behavior and cost of smart solutions) and regulatory issues (market organization to valorize the flexibility at distribution level) that will have to be addressed for a successful implementation of demand flexibility.

\section{References}

1. Walling, R.A.; Saint, R.; Dugan, R.C.; Burke, J.; Kojovic, Ljubomir A.: "Summary of Distributed Resources Impact on Power Delivery Systems," Power Delivery, IEEE Transactions on, vol.23, no.3, July 2008.

2. P. Hallberg (Chair) et al., "Active distribution system management: a key tool for the smooth integration of distributed generation," Eurelectric TF Active System Management, February 2013.

3. Sullivan, M., Bode, J.,Kellow, B.,Woehleke, S.,Eto, J.:'Using Residential AC Load Control in Grid Operations: PG\&E's Ancillary Service Pilot,' Smart Grid, IEEE Transactions on, vol.4, no.2, pp.1162,1170, June 2013.

4. P. Codani, Y. Perez, and M. Petit, "Financial shortfall for electricvehicles: Economic impacts of transmission system operators marketdesigns," Energy, vol. 113, pp. 422-431, 2016.

5. Christakou, K.,Tomozei, D.-C., Le Boudec, J.-Y.,Paolone, M.:'GECN: Primary Voltage Control for Active Distribution Networks via Real-Time Demand-Response,'Smart Grid, IEEE Transactions on , vol.5, no.2, pp.622,631, March 2014.

6. Zakariazadeh, Alireza, Homaee, Omid, Jadid, Shahram and Siano, Pierluigi,: A new approach for real time voltage control using demand response in an automated distribution system, Applied Energy, 117, issue C, p. 157-166, 2014.

7. Gill, S.; Kockar, I.; Ault, G.W., "Dynamic Optimal Power Flow for Active Distribution Networks," in Power Systems, IEEE Transactions on , vol.29, no.1, pp.121-131, Jan. 2014

8. N. Li, L. Chen and S. H. Low, "Exact convex relaxation of OPF for radial networks using branch flow model," 2012 IEEE Third International Conference on Smart Grid Communications (SmartGridComm), Tainan, 2012, pp. 7-12. 
9. M. Nick, R. Cherkaoui, J. Y. LeBoudec and M. Paolone, "An Exact Convex Formulation of the Optimal Power Flow in Radial Distribution Networks Including Transverse Components," in IEEE Transactions on Automatic Control, vol. PP, no. 99, pp. 1-1.

10. M. Farivar and S. H. Low, "Branch Flow Model: Relaxations and Convexification - Part I," IEEE Transactions on Power Systems, vol. $28,2013$.

11. S. M. de Oca, P. Belzarena and P. Monzón, "Optimal Demand Response in distribution networks with several energy retail companies," 2016 IEEE Conference on Control Applications (CCA), Buenos Aires, 2016, pp. 1092-1097.

12. W. Shi, N. Li, X. Xie, C. C. Chu and R. Gadh, "Optimal Residential Demand Response in Distribution Networks," in IEEE Journal on Selected Areas in Communications, vol. 32, no. 7, pp. 1441-1450, July 2014.

13. M. Kamgarpour et al., "Modeling options for demand side participation of thermostatically controlled loads," 2013 IREP Symposium Bulk Power System Dynamics and Control - IX Optimization, Security and Control of the Emerging Power Grid, Rethymno, 2013, pp. $1-15$.

14. T. Remani, E. A. Jasmin and T. P. I. Ahamed, "Load scheduling problems under demand response schemes: A survey," 2015 IEEE International Conference on Signal Processing, Informatics, Communication and Energy Systems (SPICES), Kozhikode, 2015, pp. 1-5.

15. H. Wang, K. Meng, Z. Y. Dong, Z. Xu, F. Luo and K. P. Wong, "Efficient real-time residential energy management through MILP based rolling horizon optimization," 2015 IEEE Power \& Energy Society General Meeting, Denver, CO, 2015, pp. 1-6.

16. N. G. Paterakis, O. Erdinç, A. G. Bakirtzis and J. P. S. Catalão, "Optimal Household Appliances Scheduling Under Day-Ahead Pricing and Load-Shaping Demand Response Strategies," in IEEE Transactions on Industrial Informatics, vol. 11, no. 6, pp. 1509-1519, Dec. 2015.

17. K. Balasubramaniam, P. Saraf, P. Hazra, R. Hadidi and E. Makram, "A MILP formulation for utility scale optimal demand side response," 2016 IEEE Power and Energy Society General Meeting (PESGM), Boston, MA, 2016, pp. 1-5.

18. E. L. Karfopoulos and N. D. Hatziargyriou, "Distributed Coordination of Electric Vehicles Providing V2G Services," in IEEE Transactions on Power Systems, vol. 31, no. 1, pp. 329-338, Jan. 2016.

19.. Siano, Pierluigi, and Debora Sarno. "Assessing the benefits of residential demand response in a real time distribution energy market." Applied Energy 161, 2016, pp. 533-551.

20. Hee-Tae Roh; Jang-Won Lee, "Residential Demand Response Scheduling With Multiclass Appliances in the Smart Grid," in Smart Grid, IEEE Transactions on, vol.7, no.1, pp.94-104, Jan. 2016

21. Ghasemi, Ahmad, Seyed Saeidollah Mortazavi, and Elaheh Mashhour. "Integration of nodal hourly pricing in day-ahead SDC (smart distribution company) optimization framework to effectively activate demand response." Energy 86 (2015): 649-660.

22. X. Fang, Q. Hu, F. Li, B. Wang and Y. Li, "Coupon-Based Demand Response Considering Wind Power Uncertainty: A Strategic Bidding Model for Load Serving Entities," in IEEE Transactions on Power Systems, vol. 31, no. 2, pp. 1025-1037, March 2016.

23. M. Ali, M. Z. Degefa, M. Humayun, A. Safdarian and M. Lehtonen, "Increased Utilization of Wind Generation by Coordinating the Demand Response and Real-time Thermal Rating," in IEEE Transactions on Power Systems, vol. 31, no. 5, pp. 3737-3746, Sept. 2016.

24. Mahmoudi, Nadali, Tapan K. Saha, and Mehdi Eghbal. "Modelling demand response aggregator behavior in wind power offering strategies." Applied Energy 133 (2014): 347-355.

25. H. Ding, Z. Hu and Y. Song, "Rolling Optimization of Wind Farm and Energy Storage System in Electricity Markets," in IEEE Transactions on Power Systems, vol. 30, no. 5, pp. 2676-2684, Sept. 2015.

26. S. Yang, D. Zeng, H. Ding, J. Yao, K. Wang and Y. Li, "Stochastic security-constrained economic dispatch for random responsive priceelastic load and wind power," in IET Renewable Power Generation, vol. 10, no. 7, pp. 936-943, 72016.

27. A. O'Connell, D. Flynn and A. Keane, "Rolling Multi-Period Optimization to Control Electric Vehicle Charging in Distribution Networks," in IEEE Transactions on Power Systems, vol. 29, no. 1, pp. 340-348, Jan. 2014.

28. Vanet, Emmanuelle. "Distributed intelligence and hierarchical approach of distributed balancing markets in Smart Grids." PhD Thesis, Grenoble Alpes University, 2016.

29. He. Y, Petit. M, "Valorization of Demand Response for Voltage Control in MV Distribution Grids with distributed generation", Proceedings of the IEEE Energycon 2016, Leuven, 2016.

30. N. Saker, M. Petit and J. L. Coullon, "Demand side management of electrical water heaters and evaluation of the Cold Load Pick-Up characteristics (CLPU)," 2011 IEEE Trondheim PowerTech, Trondheim, 2011, pp. 1-8.

31. Enedis, Opendata, www.data.enedis.fr 